\title{
The Silicon Tracking System of the CBM experiment at FAIR
}

\author{
Maksym Teklishyn ${ }^{1,2, \star}$ on behalf of the CBM Collaboration \\ ${ }^{1}$ Facility for Antiproton and lon Research in Europe GmbH (Darmstadt, Germany) \\ ${ }^{2}$ Institute for Nuclear Research (Kyiv, Ukraine)
}

\begin{abstract}
The Silicon Tracking System (STS) is the central detector in the Compressed Baryonic Matter (CBM) experiment at FAIR. Operating in the $1 \mathrm{Tm}$ dipole magnetic field, the STS will enable pile-up free detection and momentum measurement of the charged particles originating from beam-target nuclear interactions at rates up to $10 \mathrm{MHz}$. The STS consists of 8 tracking stations based on double-sided silicon micro-strip sensors equipped with fast, self-triggering read-out electronics. With about two million read-out channels, the STS will deliver a high-rate stream of time-stamped data that is transferred to a computing farm for on-line event determination and analysis. The functional building block is a detector module consisting of a sensor, micro-cables and two front-end electronics boards. In this contribution, the development status of the STS components and the system integration is discussed and an outlook on the detector construction is given.
\end{abstract}

\section{Introduction}

The Compressed Baryonic Matter (CBM) experiment aims to explore of the phase diagram of strongly interacting matter at moderate temperatures and high net baryon densities. It is planned as a part of the Facility for Antiproton and Ion Research (FAIR) complex in Darmstadt, Germany [1]. The SIS100 synchrotron will provide primary beams of protons up to $29 \mathrm{GeV}$, gold ions up to $11 \mathrm{AGeV}$ and nuclei with $Z / A=1 / 2$ up to $14 A \mathrm{GeV}$ [2].

The expected beam-target interaction rate is up to $10 \mathrm{MHz}$, thus pushing the frontier of heavy ion physics. The comparison of the anticipated CBM performance with the existing and forthcoming experiments is shown in Fig. 1, left. Such a high interaction rate is needed for the observation of particles with relatively low production cross-section (particularly multi-strange hyperons, see Fig. 1, right), sub-threshold production, and precise studies of collective effects, such as particle flow fluctuations. The required performance of the experiment challenges different systems of the detector setup. Particularly, an ultra-light, fast and precise tracking system is needed to fulfil the physics program of CBM $[2,4]$.

\section{Concept of the Silicon Tracking System}

The STS is the core tracking detector of the CBM experiment. It consists of 8 tracking stations mounted on a carbon fiber support structure; the detector will be placed in the gap of the $1 \mathrm{Tm}$ superconducting dipole magnet $30 \mathrm{~cm}$ downstream the target. The expected material budget is about

\footnotetext{
^e-mail: m.teklishyn@gsi.de
} 

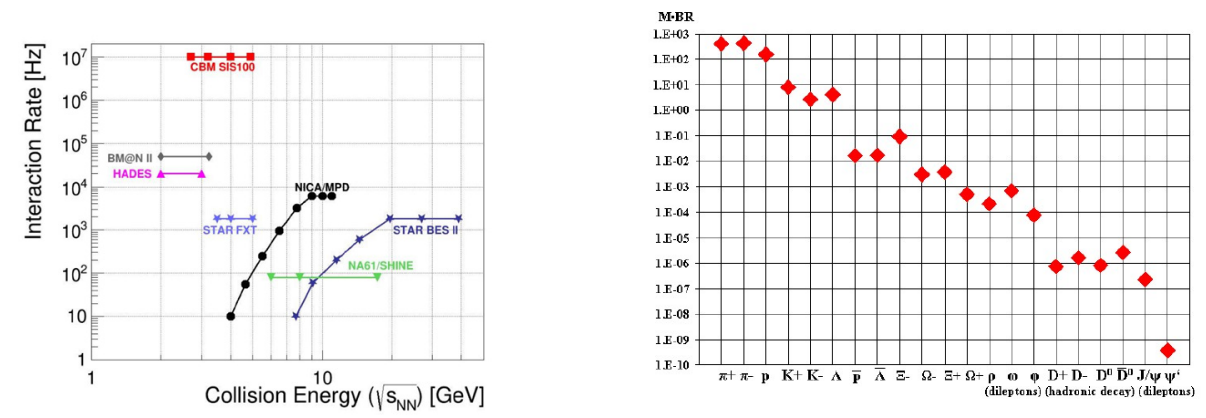

Figure 1. Left: Interaction rate with respect to the collision energy for existing and forthcoming heavy ion experiments [2]. Right: Particle multiplicities times branching ratio for central $\mathrm{Au}+\mathrm{Au}$ collisions at $25 \mathrm{AGeV}$ as calculated with the HSD transport code and the statistical model [5].

$0.3-1.6 \% X_{0}$ per station, which keeps the multiple scattering sufficiently low for the reconstruction of soft particles. In order to achieve such conditions, the read-out electronics is placed outside of the detector acceptance on aluminium cooling plates [3, 4].

Double-sided double-metal silicon micro-strip sensors are the main building elements of the STS detector. The sensors are made of $300 \mu \mathrm{m}$ thick n-type silicon wafers; they come in the following main form factors, related to four discrete strip lengths: $22 \times 62 \mathrm{~mm}^{2}, 42 \times 62 \mathrm{~mm}^{2}, 62 \times 62 \mathrm{~mm}^{2}$ and $124 \times 62 \mathrm{~mm}^{2}$, see Fig.2, right. There are 1024 strips with $58 \mu \mathrm{m}$ pitch on each side a sensor; one can see a strip pattern in Fig. 2, left. Strips on the n-side of the sensor are parallel to its edge while p-side strips are tilted by $7.5^{\circ}$ to provide the position resolution. This way the number of false track points is suppressed while the spatial resolution in horizontal or magnet bending plane remains sufficiently high. One sensor is read out by 16 STS-XYTER chips mounted on two front-end boards. The STS-XYTER chip was developed specifically for the project [10]. The read-out electronics is connected to the silicon micro-strip sensors via ultra-thin aluminium-polyimide multi-line cables [4].
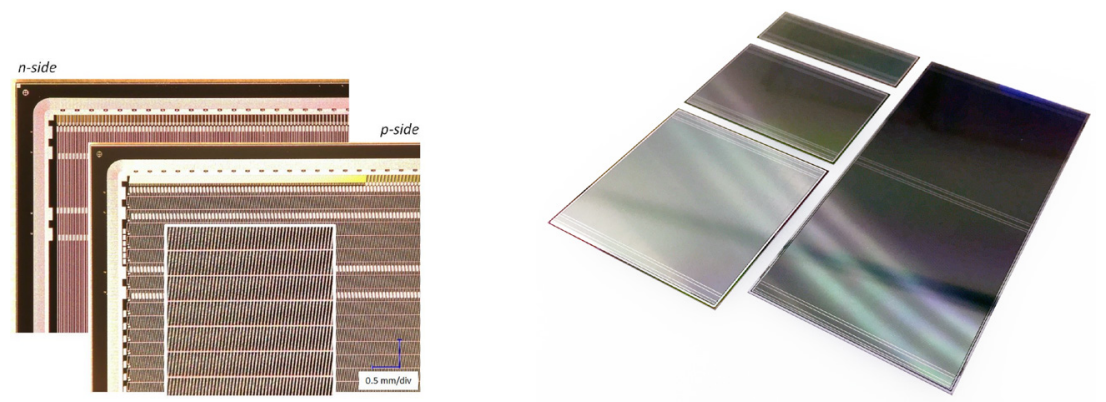

Figure 2. Left: Close-up views of prototype silicon micro-strip sensors. Right: Prototype sensors produced in the four main shapes.

For the dissipation of the heat produced by the read-out electronics a dedicated bi-phase $\mathrm{CO}_{2}$ cooling system is being developed. The temperature of the entire system will be kept below $-5^{\circ} \mathrm{C}$ to limit the effect of the sensor bulk damage due to the radiation conditions: the lifetime equivalent 
$1 \mathrm{MeV}$ neutron flux reaches $10^{14} \mathrm{~cm}^{-2}$ in the inner most region of the tracker [7, 8]. By cooling sensors we expect to limit the leakage current through the sensor bulk in order to suppress the shot noise. Numeric simulations of the heat transfer as well as measurements with prototypes are being actively performed by STS team [6,9].

The STS detector system meets various constraints regarding its compactness and complexity; the STS engineering team responds this challenges by developing dedicated integration solutions.

\section{System integration}

The STS system integration focuses on a detector that can be mounted in the gap of the dipole magnet and be extracted for maintenance. The current activities address the structure of the system, the space requirements of mechanical STS components with technical solutions for their installation, positioning precision, the cooling of electronics and routing the supplies taking into account the thermal enclosure [6].

Full-scale prototypes of the important detector parts are being designed by the engineering team of the STS project. In Fig. 3, left, one can see the current CAD design of a quarter unit of the largest station with the relevant design features; Fig. 3, right, depicts a process of the ladder mounting on the prototype.
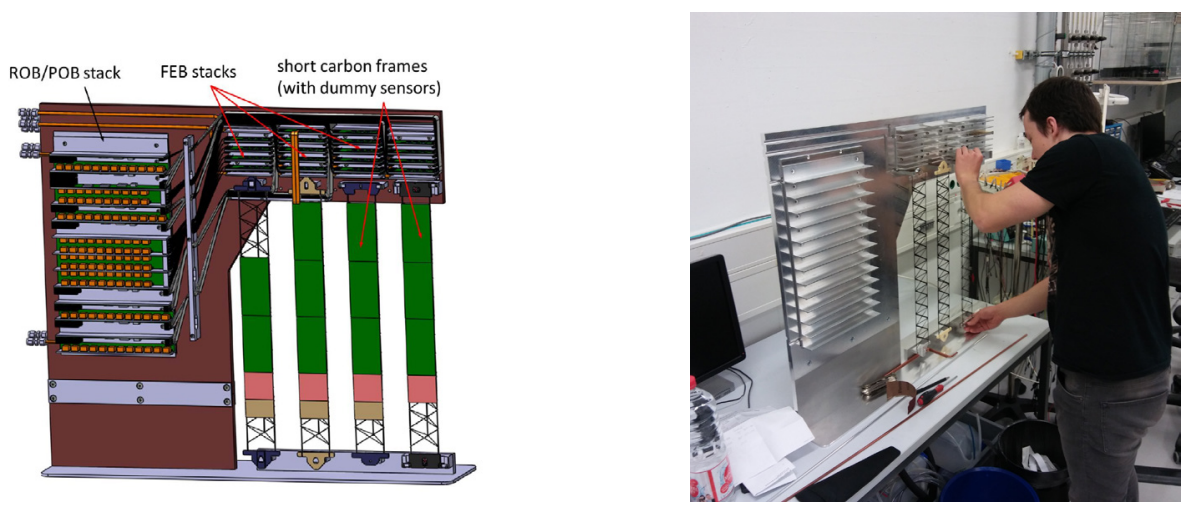

Figure 3. Left: Drawing of a prototype quarter unit. Right: A photo of the process of the ladder mounting.

Also a preliminary analysis of the cable routing was performed using dedicated tool boxes of the CAD software. Model verification based on the work with real assembly are current tasks addressed by the team. Other activities are related to the development and testing of tools for the ladder assembly, tab-bonding of the micro-cables to the sensors and electronics, development of the cooling system and thermal enclosure of the STS setup [6].

Since individual components can not be dismounted after being integrated to the setup, extensive quality assurance procedures are required on each step in order to obtain the anticipated performance of the system.

\section{Quality Assurance of the components}

Several Quality Assurance (QA) centres are being established providing evaluation of the the key components of the STS setup: silicon sensors, micro-cables end electronics. After assembly, the equipped modules are subjected to additional procedures verifying their overall performance. 

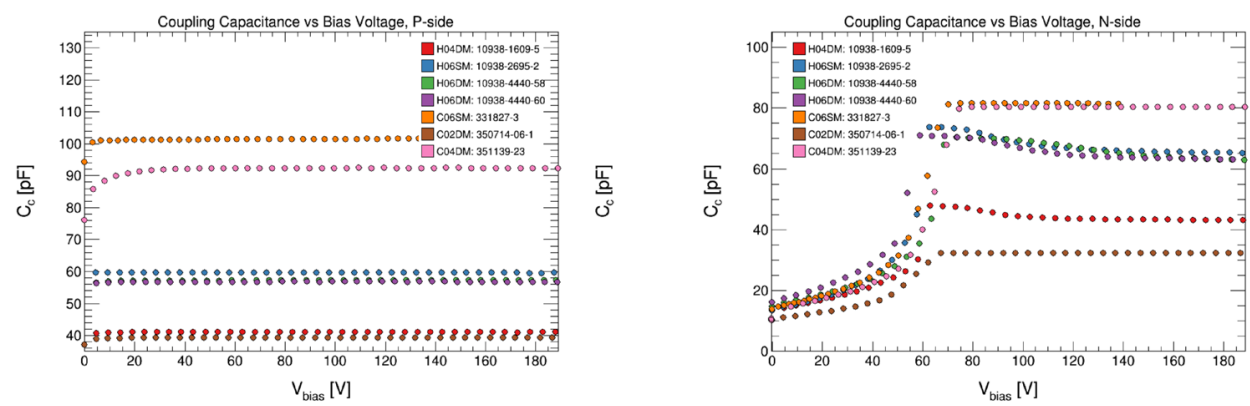

Figure 4. Coupling capacitance as function of the bias voltage for the p-side (left) and n-side (right) of $62 \times$ $62 \mathrm{~mm}^{2}$ sensors measured with the needle probe station at Tübingen University.

The main tools for testing the components are: needle probe stations in GSI, Darmstadt and Tübingen University, allowing to measure electrical characteristics of sensors and their individual strips (see Fig 4 for the example of such tests) [11], an optical inspection stand in Tübingen for sensor and microcable QA, and a setup for the radioactive source tests in GSI.

\section{Conclusions}

The STS detector is being developed by a team of international participants from different institutions. For the moment the main focus is on integration questions, the development of QA procedures for the component mass-production stage, and the preparation towards the production readiness with a set of tests, including radioactive source measurements in the laboratory as well as relativistic proton beam tests [12].

The detector is planned to be produced and installed in the CBM cave by 2021 .

\section{References}

[1] H. H. Gutbrod et al. (Eds.) FAIR Baseline Technical Report, ISBN 3-9811298-0-6 Nov. 2006.

[2] T. Ablyazimov et al. [CBM Collaboration], Eur. Phys. J. A 53 (2017) no.3, 60

[3] J. M. Heuser [CBM Collaboration], Acta Phys. Polon. Supp. 9 (2016) 221.

[4] J. M. Heuser et al. Darmstadt, GSI, GSI-2013-05499, GSI Report 2013-4, 167 p., 2013

[5] B. Friman et al. (Eds.), The CBM Physics Book, Lect. Notes Phys. 814, Springer 2011, p. 639

[6] I. Selyuzhenkov, A. Toia, Darmstadt, GSI, GSI-2017-00564, 2017

[7] I. Momot, M. Singla, M. Teklishyn, V. Pugatch and J. Heuser JPCS 668 (2016) no.1, 012117

[8] M. Singla, P. Larionov, T. Balog et al. 2016, Nucl. Instr. Meth. Phys. Res. A, 824, 462

[9] V. Friese, C. Sturm, A. Toia, Darmstadt, GSI, GSI-2016-01450, 2016

[10] K. Kasinski, R. Kleczek and R. Szczygiel, JINST 11 (2016) no.02, C02024.

[11] P. Larionov 2016, Journal of Instrumentation, 11, C01033

[12] M. Teklishyn [CBM Collaboration], PoS(BORMIO2017)055 